\title{
Exploring the behaviour of long gamma-ray bursts using an intrinsic multi-wavelength afterglow correlation
}

\author{
S. R. Oates ${ }^{* a, b}$, J. L. Racusin ${ }^{c}$, M. De Pasquale ${ }^{b, d}$, M. J. Page ${ }^{b}$, A. J. Castro-Tirado ${ }^{a, e}$, \\ J. Gorosabel ${ }^{a, f, g}$, P. J. Smith ${ }^{b}$, A. A. Breeveld ${ }^{b}$, N. P. M Kuin ${ }^{b}$ \\ ${ }^{a}$ Instituto de Astrofísica de Andalucía (IAA-CSIC), Glorieta de la Astronomía s/n, E-18008, \\ Granada, Spain \\ ${ }^{b}$ Mullard Space Science Laboratory, University College London, Holmbury St. Mary, Dorking \\ Surrey, RH5 6NT, UK \\ ${ }^{c}$ Astrophysics Science Division, NASA Goddard Space Flight Center, 8800 Greenbelt Road, \\ Greenbelt, Maryland 20771, USA \\ ${ }^{d}$ Istituto Astrofisica Spaziale Fisica Cosmica, Palermo, Italy \\ ${ }^{e}$ Unidad Asociada Departamento de Ingeniería de Sistemas y Automática, E.T.S. de Ingenieros \\ Industriales, Universidad de Málaga, Spain \\ ${ }^{f}$ Unidad Asociada Grupo Ciencias Planetarias UPV/EHU-IAA/CSIC, Departamento de Física \\ Aplicada I, E.T.S., Ingeniería, Universidad del País Vasco UPV/EHU, Bilbao, Spain \\ ${ }^{g}$ Ikerbasque, Basque Foundation for Science, Bilbao, Spain \\ E-mail: srodiaa.es
}

We have discovered an intrinsic correlation between the early optical/UV afterglow luminosity ( $\log \mathrm{L}_{200 \mathrm{~s}}$, measured at restframe 200s) and average optical afterglow decay rate $(\alpha>200$ s, measured from restframe $200 \mathrm{~s}$ onwards) of long duration Gamma-ray Bursts (GRBs) ([3]). In these proceedings we determine that the luminosity-decay correlation is also observed in the X-ray light curves and we explore how this correlation is related to the prompt emission phase, specifically with the parameters: the isotropic energy and the restframe $\mathrm{T}_{90}$ parameter (duration over which $90 \%$ of the emission is observed). We begin by examining the relationships predicted by the standard afterglow model. It is not easy to analytically predict observed correlations between two parameters, since a sample is likely to consist of GRB afterglows satisfying different spectral regimes. Therefore we use a Monte Carlo simulation to predict the relationships we should observe with our sample of 48 GRBs. We then use the sample of X-ray and optical/UV afterglow light curves to explore the observed relationships between two parameters. While some observed correlations are consistent with the simulations, correlations between $\log \mathrm{L}_{200 \mathrm{~s}}$ and $\alpha_{>200 \mathrm{~s}}$, or $\log \mathrm{E}_{\mathrm{iso}}$ and $\alpha_{>200 \mathrm{~s}}$ are inconsistent with simulations. This suggests that while the basic afterglow model can potentially explain some of the observed correlations, it can not explain all of them. We briefly mention alternative more complex models.

Swift: 10 Years of Discovery,

2-5 December 2014

La Sapienza University, Rome, Italy

* Speaker. 


\section{Introduction}

GRB afterglow luminosity light curves cluster and it is apparent that the luminosity distribution is wider during the early part of the afterglow and becomes narrower as the afterglows fade. This suggests that the most luminous GRB afterglows at early epochs, may decay more quickly than less luminous afterglows. We tested this hypothesis, with a sample of optical/UV light curves, using the logarithmic brightness (measured at rest frame 200s), $\log \mathrm{L}_{200 \mathrm{~s}}$ and average decay rate of GRB afterglows (measured from rest frame 200s onwards), $\alpha_{>200 s}$. A Spearman rank correlation gives a coefficient of -0.58 at a significance of $4.2 \sigma$ ([3]). Thus confirming our observation that the brightest GRB afterglows, in the optical/UV, decay more quickly than the less luminous afterglows. In the following, we further explore the $\log \mathrm{L}_{200 \mathrm{~s}}-\alpha_{>200 \mathrm{~s}}$ relation. In these proceedings, we show that the correlation is also observed in the X-rays. We examine how the parameters in each correlation relate to each other and how they relate to the parameters of the prompt emission phase, namely the isotropic energy $\mathrm{E}_{\text {iso }}$ and the duration over which $90 \%$ of the prompt emission was observed $\mathrm{T}_{90}$. We will also determine if our sample results in correlations consistent with those predicted by the standard afterglow model using a Monte Carlo simulation. For each GRB, we used interpolation between restframe 100 and 2000s to obtain the optical luminosity at restframe $200 \mathrm{~s}$ and for the X-ray we measured the luminosity at restframe $200 \mathrm{~s}$ from the best fit light curve model. To obtain the average decay rate, we fit a single power-law to each optical and X-ray light curve using data from restframe $200 \mathrm{~s}$ onwards. We note that for 8 GRBs the X-ray luminosity at restframe $200 \mathrm{~s}$ is likely contaminated by the end of the prompt emission. In these situations the average decay index is measured using data beyond restframe $200 \mathrm{~s}$ that is not dominated by the prompt emission. In these cases, we extrapolate from the best fit afterglow model the decay index of the segment closest to restframe $200 \mathrm{~s}$ and measure an estimate of the luminosity at restframe $200 \mathrm{~s}$ from this extrapolation (see [4] for further details). To determine the degree of correlation and the relationship between these and other parameters we use both Spearman rank correlation and linear regression.

\section{Monte Carlo Simulation}

In the standard afterglow model, depending on the properties and environment of the GRB there is usually more than one relationship to describe how two parameters are related (e.g [1, 2]). This therefore makes a simple analytic prediction of the expected relationships in a sample of observed parameters difficult to determine. We therefore use a Monte Carlo simulation to produce, $10^{4}$ times, a sample of 48 GRBs. For each simulated sample, we performed linear regression and we also calculated the Spearman rank coefficient between several parameters: the optical/UV and $\mathrm{X}$-ray luminosities, the optical/UV and X-ray decay indices and $\mathrm{E}_{\mathrm{iso}}$. From the results we obtained predictions for Spearman rank coefficients and the relationships between each pair of parameters, for an observed sample of 48 GRB afterglows. The predictions are given in Table 1.

\section{Results \& Discussion}

A correlation is observed between $\log \mathrm{L}_{200 \mathrm{~s}}$ and $\alpha_{>200 \mathrm{~s}}$ for both the X-ray and optical/UV light curves (see also [4]. The Spearman rank coefficients and best fit linear regressions are given 


\begin{tabular}{llcrc}
\hline \multicolumn{2}{c}{ Parameters } & $\begin{array}{c}\text { Simulated Spearman } \\
\text { Rank Coefficient }\end{array}$ & $\begin{array}{c}\text { - Best fit linear regression for simulation- } \\
\text { Slope }\end{array}$ & Constant \\
\hline \hline $\log \mathrm{L}_{\mathrm{O}, 200 \mathrm{~s}}$ & $\log \mathrm{L}_{\mathrm{X}, 200 \mathrm{~s}}$ & $0.92 \pm 0.02$ & $0.82 \pm 0.04$ & $3.76 \pm 1.25$ \\
$\alpha_{O,>200 \mathrm{~s}}$ & $\alpha_{X,>200 \mathrm{~s}}$ & $0.74 \pm 0.06$ & $1.10 \pm 0.15$ & $-0.04 \pm 0.17$ \\
$\log \mathrm{L}_{\mathrm{O}, 200 \mathrm{~s}}$ & $\alpha_{O,>200 \mathrm{~s}}$ & $-0.30 \pm 0.14$ & $-0.04 \pm 0.02$ & $0.31 \pm 0.65$ \\
$\log \mathrm{L}_{\mathrm{X}, 200 \mathrm{~s}}$ & $\alpha_{X,>200 \mathrm{~s}}$ & $-0.20 \pm 0.14$ & $-0.04 \pm 0.03$ & $0.10 \pm 0.78$ \\
$\log \mathrm{E}_{\text {iso }}$ & $\alpha_{O,>200 \mathrm{~s}}$ & $-0.06 \pm 0.15$ & $-0.03 \pm 0.06$ & $0.32 \pm 2.91$ \\
$\log \mathrm{E}_{\text {iso }}$ & $\alpha_{X,>200 \mathrm{~s}}$ & $-0.09 \pm 0.15$ & $-0.04 \pm 0.06$ & $0.76 \pm 3.13$ \\
$\log \mathrm{E}_{\text {iso }}$ & $\log \mathrm{L}_{\mathrm{O}, 200 \mathrm{~s}}$ & $0.51 \pm 0.11$ & $4.43 \pm 1.02$ & $-200.76 \pm 37.33$ \\
$\log \mathrm{E}_{\text {iso }}$ & $\log \mathrm{L}_{\mathrm{X}, 200 \mathrm{~s}}$ & $0.54 \pm 0.11$ & $3.28 \pm 0.71$ & $-142.22 \pm 21.57$ \\
\hline
\end{tabular}

Table 1: The Spearman rank coefficient and linear regression parameters as predicted by the synchrotron model for a sample of 48 GRBs. These values were computed with a Monte Carlo simulation with $10^{4}$ trials.

\begin{tabular}{|c|c|c|c|c|c|c|c|}
\hline \multicolumn{2}{|c|}{ Parameters } & \multirow{2}{*}{$\begin{array}{c}\text { Spearman Rank } \\
\text { Coefficient }\end{array}$} & \multirow{2}{*}{$\begin{array}{c}\text { Null } \\
\text { Hypothesis }\end{array}$} & \multirow{2}{*}{$\begin{array}{c}\text { Partial } \\
\text { Spearman Rank }\end{array}$} & \multirow{2}{*}{$\begin{array}{c}\text { Null } \\
\text { Hypothesis }\end{array}$} & \multicolumn{2}{|c|}{ —Best fit linear regression- } \\
\hline$x$-axis & $y$-axis & & & & & Slope & Constant \\
\hline $\log \mathrm{L}_{\mathrm{O}, 200 \mathrm{~s}}$ & $\alpha$ & $-0.58(0.11)$ & $1.90 \times 10^{-5}$ & -0.50 & $2.85 \times 10^{-4}$ & $-0.28 \pm 0.04$ & $7.72 \pm 1.31$ \\
\hline $\log \mathrm{L}_{\mathrm{X}, 200 \mathrm{~s}}$ & $\alpha_{X,>200 \mathrm{~s}}$ & $-0.69(0.09)$ & $8.03 \times 10^{-8}$ & -0.63 & $1.58 \times 10^{-6}$ & $-0.26 \pm 0.05$ & $6.71 \pm 1.39$ \\
\hline
\end{tabular}

Table 2: The observed relationships between $\log \mathrm{L}_{200 s}-\alpha_{>200 \mathrm{~s}}$ in the optical/UV and X-ray: the Spearman rank coefficient, $1 \sigma$ error and null hypothesis; the coefficient of the partial Spearman rank and null hypothesis, which tests the correlation between two parameters taking into account the parameters dependence on redshift; the slope and constant values provided by the best fit linear regression.

in Table 2. The linear regressions are consistent at $1 \sigma$. This suggests that the same mechanism is producing the relationship between the luminosity at restframe $200 \mathrm{~s}$ and the average decay rate from restframe $200 \mathrm{~s}$ for both the X-ray and optical/UV light curves. Comparing the linear regression parameters for the observed and predicted data, we find that the slopes and constant parameters are inconsistent at $\gtrsim 4 \sigma$. Therefore this implies that correlations as strong as those observed, between $\log \mathrm{L}_{200 \mathrm{~s}}$ and $\alpha_{>200 \mathrm{~s}}$ are not expected in the standard afterglow model.

We find, and as predicted by the standard afterglow model, the brightest afterglows in the optical/UV are the brightest in the X-ray and there is a relationship between the average decay indices (Fig 1 top panels). In the top right panel we also show the expected relationships between the decay indices in terms of the standard afterglow model for the optical/UV and X-ray light curves. The best fit regression line, lies above, but close to, the line $\alpha_{X,>200 \mathrm{~s}}=\alpha_{O,>200 \mathrm{~s}}-0.25$. This implies that there is unlikely to be a single closure relation responsible for producing the X-ray and optical afterglows, although a constant density medium is preferred and the cooling frequency is likely between the X-ray and optical/UV bands at least for a large number of events. This is consistent with recent analysis by [6], [7], [8] and [9]. We find that both relationships, between the $\mathrm{X}$-ray and optical/UV luminosities and the decay indices are consistent with the simulations.

The standard afterglow model predicts a relationship between the isotropic energy $\mathrm{E}_{\text {iso }}$, and the luminosity of the afterglow. However our observed data (Fig 1 middle panels) predicts a Spearman rank correlation slightly stronger in comparison to that found for the simulation, with only $0.3 \%$ and $0.06 \%$ of the simulations having Spearman rank coefficients equal to or larger than that observed for the optical/UV and X-ray, respectively. This suggests that, the observed relationships are slightly more tightly correlated than expected from the standard afterglow model. This is likely related to our choice of efficiency in the simulation. A wide range in efficiency is likely to introduce 
more scatter in the relationship between $\log \mathrm{E}_{\text {iso }}$ and $\log \mathrm{L}_{200 \mathrm{~s}}$. The results of the linear regression indicate that the observed relationship is shallower than that predicted and are inconsistent at $\gtrsim 3 \sigma$.

In the observed sample we also find relationships between $\log \mathrm{E}_{\text {iso }}$ and the optical and X-ray decay indices (Fig 1 bottom panels). In the simulations we find that only $0.01 \%$ predict a relationship the same or stronger than what we observe between $\log \mathrm{E}_{\mathrm{iso}}$ and $\alpha_{\mathrm{X},>200 \mathrm{~s}}$ and $0.03 \%$ predict similar or stronger relationship between $\log \mathrm{E}_{\mathrm{iso}}$ and $\alpha_{\mathrm{O},>200 \mathrm{~s}}$. This and linear equations inconsistent with simulations at $\gtrsim 2.2 \sigma$, implies that these correlations are not predicted by the standard afterglow model. However, since we find correlations between $\log \mathrm{E}_{\mathrm{iso}}, \alpha_{>200 \mathrm{~s}}$ and $\log \mathrm{L}_{200 \mathrm{~s}}$, this suggests that what happens during the prompt phase has direct effect on the afterglow.

We also explored relationships between the duration of the $\gamma$-ray emission in the restframe, $\mathrm{T}_{90}$, with $\log \mathrm{L}_{200 \mathrm{~s}}, \alpha_{>200 \mathrm{~s}}$ and $\log \mathrm{E}_{\text {iso }}$. Significant correlations are not found amongst these parameters using the entire sample of 48 GRBs. This is consistent with the findings of [5]. However, as part of our checks, we repeated our analysis for all pairs of parameters excluding the 8 GRBs for which the X-ray light curves were contaminated at 200s by the tail of the prompt emission. We found that the results do not significantly change for any correlation except for those involving the restframe $T_{90}$ parameter. In these cases, we find that the Spearman rank coefficients are larger than those determined with the same analysis performed on the entire GRB sample. For three pairs of parameters restframe T90 and $\log \mathrm{L}_{\mathrm{O}, 200 \mathrm{~s}}$, restframe T90 and $\log \mathrm{E}_{\mathrm{iso}}$, and restframe T90 with $\log \mathrm{E}_{\text {peak }}$, the significance of correlation implied by Spearman rank is $>3 \sigma$ and the coefficient suggests strong correlations. However, this is most likely a selection effect. In order to observe the tail of the prompt emission at restframe 200s, the prompt emission duration should be long, but also it has to be bright or the afterglow weak so that the prompt emission can be observed above the afterglow. For these $8 \mathrm{GRBs}$ it is the chance combination of low afterglow luminosity and long duration prompt emission, which allows the tail of the prompt emission to dominate over the afterglow. Therefore these light curves cluster at large restframe T90 and low log $\mathrm{L}_{\mathrm{X}, 200 \mathrm{~s}}$. Also since $\log \mathrm{L}_{\mathrm{X}, 200 \mathrm{~s}}$ correlates with $\log \mathrm{L}_{\mathrm{O}, 200 \mathrm{~s}}$ and $\log \mathrm{E}_{\mathrm{iso}}$, we should also find clustering of these parameters with restframe T90. Examining the corresponding panels of Fig. 2, we find that the 8 GRBs are clustered in the bottom right of these panels. Therefore by removing these GRBs and repeating the correlations we are artificially inducing correlations between these parameters.

\section{Conclusions}

We found significant correlations between the X-ray and optical luminosities ( $\log \mathrm{L}_{\mathrm{O}, 200 \text { s }}$, $\left.\log \mathrm{L}_{\mathrm{X}, 200 \mathrm{~s}}\right)$ and the optical/UV and X-ray decay indices $\left(\alpha_{O,>200 \mathrm{~s}}\right.$ and $\left.\alpha_{X,>200 \mathrm{~s}}\right)$ and we also find correlations between these parameters and the isotropic energy $\left(\log \mathrm{E}_{\mathrm{iso}}\right)$. All these correlations are consistent with the idea that there is a common underlying physical mechanism, producing GRBs and their afterglows regardless of their detailed temporal behaviour.

We explored the relationships between several afterglow and prompt emission parameters and compared these to those predicted with our Monte Carlo simulation in order to determine whether the observed correlations are consistent with those predicted by the standard afterglow model. We determined that relationships between the luminosities in both the X-ray and optical/UV bands, between the decay indices and between the luminosities and the isotropic energy are predicted by the simulation of the standard afterglow model, although the slope of the relationships between lu- 

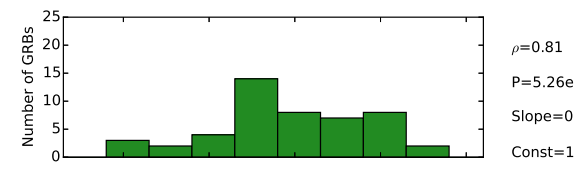

$\mathrm{P}=5.26 \mathrm{e}-12$

Slope $=0.91 \pm 0.22$

Const $=1.04 \pm 6.90$
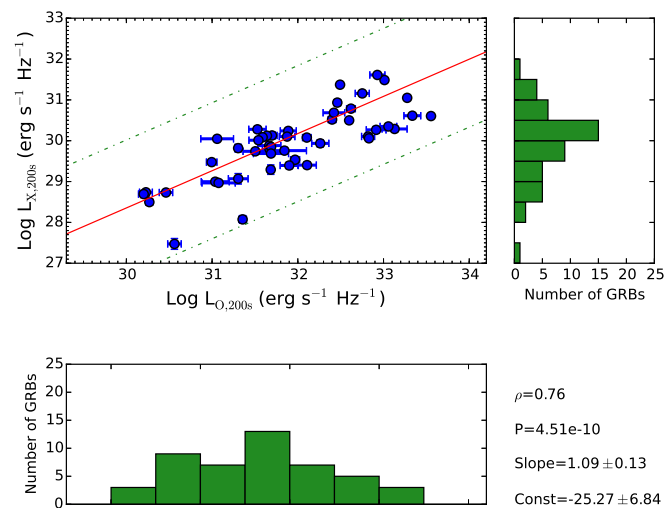

$\rho=0.76$

$\mathrm{P}=4.51 \mathrm{e}-10$

Slope $=1.09 \pm 0.13$

Const $=-25.27 \pm 6.84$
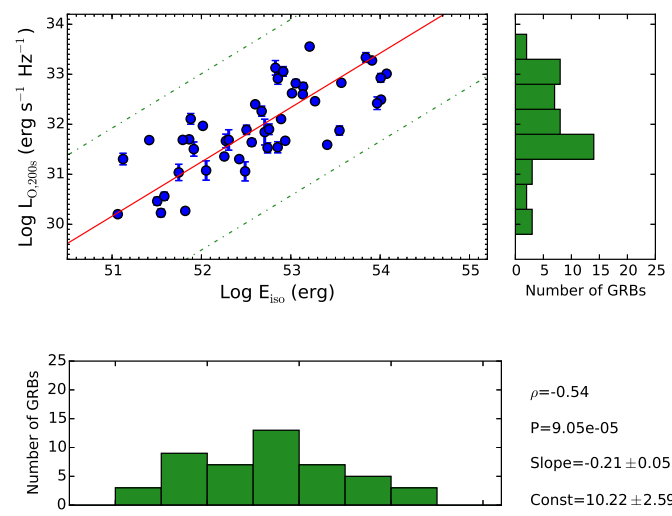

$\rho=-0.54$

$P=9.05 e-05$

Slope $=-0.21 \pm 0.05$

Const $=10.22 \pm 2.59$
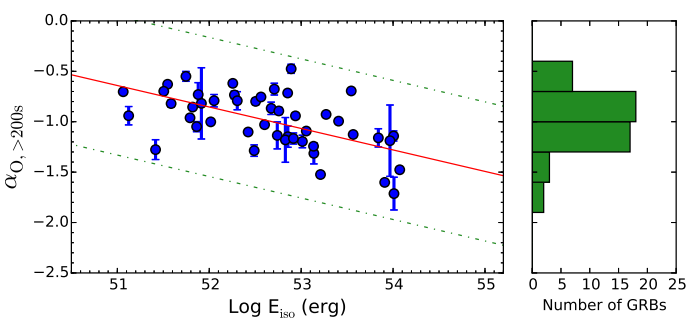
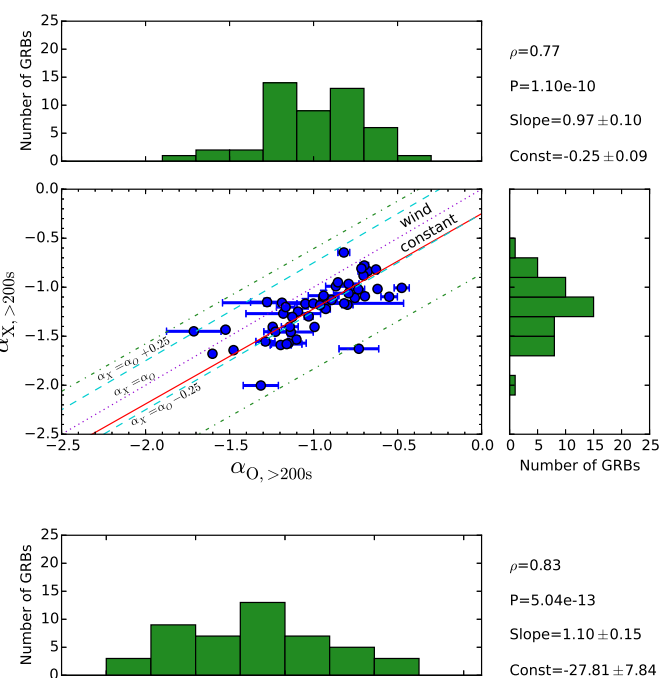

$=0.83$

$P=5.04 \mathrm{e}-13$

Slope $=1.10 \pm 0.15$

Const $=-27.81 \pm 7.84$
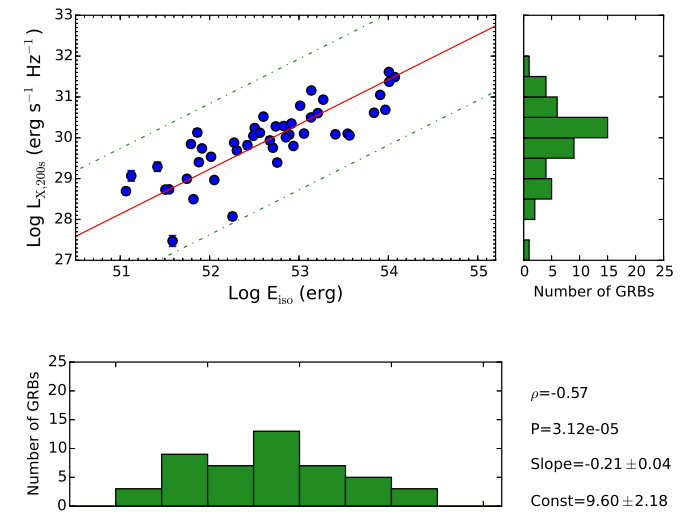

$\rho=-0.57$

$P=3.12 \mathrm{e}-05$

Slope $=-0.21 \pm 0.04$

Const $=9.60 \pm 2.18$
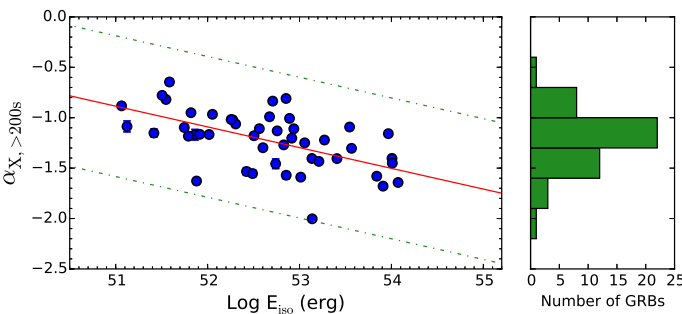

Figure 1: Top Left: Optical/UV and X-ray luminosity determined at restframe $200 \mathrm{~s}$. Top Right: Average decay rate of the optical/UV and X-ray light curves determined from restframe $200 \mathrm{~s}$ onwards. Middle Left: The optical luminosity at restframe $200 \mathrm{~s}$ versus isotropic energy. Middle Right: The X-ray luminosity at restframe $200 \mathrm{~s}$ versus isotropic energy Bottom Left: The optical average decay index determined from restframe $200 \mathrm{~s}$ versus isotropic energy. Bottom Right: The X-ray average decay index determined from restframe $200 \mathrm{~s}$ versus isotropic energy. In all panels, the red solid line represents the best fit regression and the green dot-dash line represents the $3 \sigma$ root mean square (RMS) deviation. In the top right panel, we also show relationships expected between the optical/UV and X-ray light curves from the GRB closure relations. The purple dotted line represents the optical/UV and X-ray decay indices being equal. The light blue dashed lines represent the X-ray temporal index equal to the optical/UV temporal index \pm 0.25 . In the top right corner of each panel, we give the Spearman rank coefficient, $\rho$, and corresponding null hypothesis probability, $P$, and we provide the best fit slope and constant determined by linear regression. 

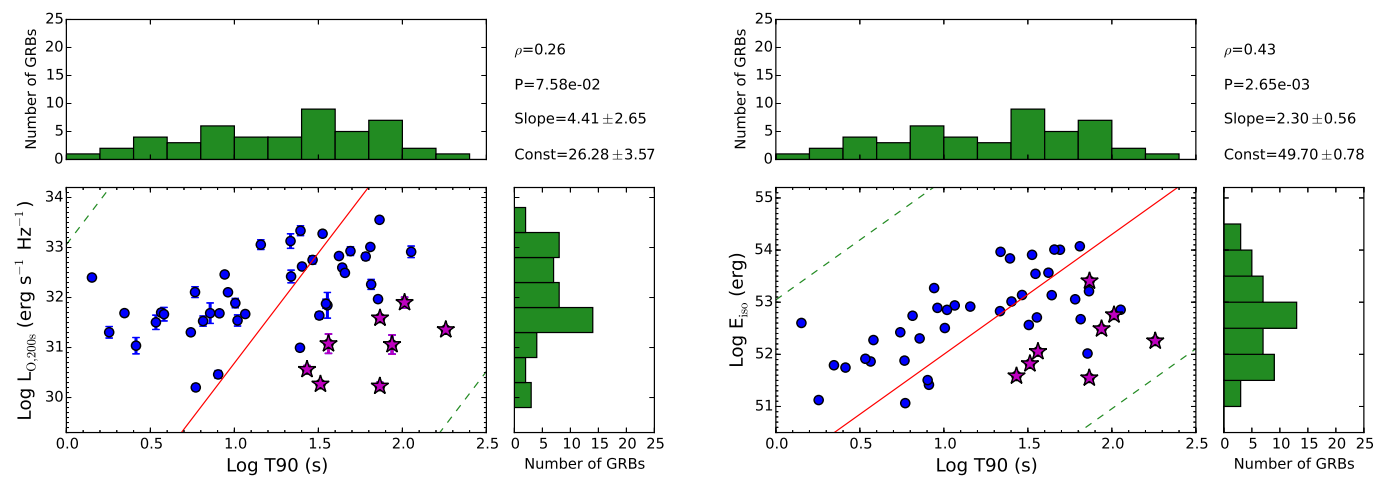

Figure 2: Left: The optical luminosity at restframe $200 \mathrm{~s}$ versus restframe $\mathrm{T}_{90}$; Right: The isotropic prompt emission $\mathrm{E}_{\text {iso }}$ versus restframe $\mathrm{T}_{90}$. The purple stars indicate those GRBs for which the X-ray afterglow was contaminated by the end of the prompt emission. All points are included when determining the best fit linear regression and Spearman rank values given in the figure. Key is otherwise the same as Fig. 1.

minosity and isotropic energy are steeper in the simulations than observed. However, the observed relationship involving the average decay indices with either luminosity at $200 \mathrm{~s}$ or the isotropic energy are not consistent with the simulations. This suggests that the observed relationships, for both the X-ray and optical/UV samples involving $\alpha_{>200 \mathrm{~s}}$ are not expected in the standard afterglow model, we therefore suggest that a more complex afterglow or outflow model is required to produce all the observed correlations. This may be due to either a viewing angle effect or by some mechanism or physical property controlling the energy release within the outflow.

\section{References}

[1] R. Sari et al., Spectra and Light Curves of Gamma-Ray Burst Afterglows, ApJL 497 (1998) L17

[2] Panaitescu, A. \& Kumar, P., Analytic Light Curves of Gamma-Ray Burst Afterglows: Homogeneous versus Wind External Media, ApJ 543 (2000) 66

[3] Oates, S. R., et al., A correlation between the intrinsic brightness and average decay rate of Swift/UVOT gamma-ray burst optical/ultraviolet light curves MNRAS 426 (2012) L86

[4] Racusin, J. L., et al., A correlation between the intrinsic brightness and average decay rate of gamma-ray burst x-ray afterglow light curves, to be submitted ApJ 2015

[5] Margutti, R., et al., The prompt-afterglow connection in gamma-ray bursts: a comprehensive statistical analysis of Swift X-ray light curves, MNRAS 428 (2013) 729

[6] Rykoff, E. S.., et al., Looking Into the Fireball: ROTSE-III and Swift Observations of Early Gamma-ray Burst Afterglows, ApJ 702 (2009) 489

[7] Oates, S. R., A statistical comparison of the optical/UV and X-ray afterglows of gamma-ray bursts using the Swift Ultraviolet Optical and X-ray Telescopes, MNRAS 412 (2011) 561

[8] Schulze, S., et al., The circumburst density profile around GRB progenitors: a statistical study, A\&A 526 (2011) A23

[9] De Pasquale, M., Physical properties of rapidly decaying Afterglows, EAS Publications Series, 61 (2013) 217 